\title{
LOS CONCEPTOS DE ONEROSIDAD Y GRATUIDAD EN EL Código Civil CHILENo
}

[The Concepts of Onerousness and Gratuitousness in the Chilean Code of Civil Procedure]

\author{
Francisca Leitao Álvarez-SalamancA* \\ Universidad de los Andes, Santiago, Chile
}

\section{RESUMEN}

Los conceptos de lo oneroso y lo gratuito en materia civil, recogidos en el artículo 1440 CC., se hallan establecidos para los contratos. En este trabajo, que recién está en su primera etapa, nos proponemos, en primer lugar, realizar una reseña histórica acerca de la fuente del mencionado artículo; $y$, en seguida, indagar algunos puntos del estado de la cuestión en la doctrina nacional sobre dichos conceptos, la cual parece mostrar abundante variedad de entendimientos.

Palabras clave

Onerosidad - Gratuidad.

\section{ABSTRACT}

The concepts of onerousness and gratuitousness in civil matters related to contracts are established in article 1440 of the Code of Civil Procedure. In this work, which is in its first stage, we initially propose to carry out an historical overview on the sources of the aforementioned article; and, then, to seek for some points of the state of affairs in the Chilean doctrine on said concepts, which seems that can be understood from different standpoints.

\section{KeYWORDS}

Onerousness - Gratuitousness.

RECIBIDO el 31 de agosto y ACEPTADO el 7 de diciembre de 2015

* Doctora en derecho por la Pontificia Universidad Católica de Valparaíso, Chile; Profesora investigadora en la Facultad de Derecho de la Universidad de los Andes. Dirección postal: Monseñor Álvaro del Portillo N ${ }^{\circ}$ 12.455, Las Condes, Santiago, Chile. Correo electrónico: franciscaleitao@uandes.cl Este trabajo forma parte de una investigación más amplia perteneciente a FONDECYT POSTDOCTORAL N³150331 de la que la autora es investigadora responsable. 


\section{INTRODUCCIÓN}

La definición de lo oneroso y gratuito en nuestro ordenamiento viene dada en el artículo 1440 CC. relativo a los contratos. De acuerdo a esta norma, la diferencia entre un contrato gratuito y uno oneroso radica en la noción de "utilidad" que debe existir entre las partes; así cuando exista sólo para una, entiende que el contrato es gratuito o de beneficencia; y si la haya para ambos, entonces el contrato es oneroso. En este trabajo nos proponemos los siguientes objetivos: $i$ ) realizar una reseña histórica acerca de la fuente del artículo 1440; y ii) indagar el estado de la cuestión en la doctrina nacional sobre algunos puntos concernientes a los conceptos de gratuito y oneroso.

Esta investigación se encuentra recién en su inicio; es por ello que dejaremos para más adelante el concepto mismo de "utilidad" tema crucial que según el artículo 1440 hace la distinción entre ambos conceptos.

\section{RESEÑA HISTÓRICA ACERCA DE LA FUENTE DEL ARTÍCULO 1440 CC.}

\section{Lo oneroso y lo gratuito en la ciencia jurídica moderna.}

Antes de entrar en la génesis del artículo 1440 CC. en una perspectiva de su codificación, es necesario advertir que la ciencia jurídica moderna tiene como característica más importante su tendencia a la abstracción y a la generalización del sistema derivada del derecho romano y del ius commune ${ }^{1}$. Esto se dio a través de las corrientes humanistas y, posteriormente, con el iusnaturalismo racionalista, hasta la llegada de los primeros códigos en la segunda mitad del siglo XVIII. Dicho rápidamente, éste fue el ambiente que sirvió de génesis a la categoría de contratos gratuitos y onerosos en la codificación, la cual comienza en la sistemática de los "actos de los hombres que tienden a la utilidad de otros hombres" elaborada por Grocio (1583-1645)2 ; en definitiva los actos pueden ser, por un lado, benéficos $y$, por otro, permutatorios. Éstos últimos son contratos onerosos (los

${ }^{1}$ Véase GuZMÁn Brito, Alejandro, Acto, negocio, contrato y causa en la tradición del derecho europeo e iberoamericano (Navarra, 2005), pp. 343-354.

${ }^{2}$ Ibíd., p. 344: "[...] No bien defina correctamente qué sea un contrato lucrativo, caracteriza a los onerosos como aquellos en los que ambas partes adquieren obligaciones, con lo cual, en realidad, define al contrato bilateral. Como es sabido, éste tanto puede ser lucrativo o gratuito, como el mandato gratuito, cuanto oneroso, como la compraventa. [...]”. Luis de Molina (1535-1600) menciona, pues, la distinción entre lucrativo y oneroso, pero la confunde, como ha dicho Guzmán, con la de unilaterales y bilaterales; éste error de Molina se entiende en cuanto tal distinción en la ciencia jurídica de su época la contraposición entre oneroso y gratuito no debe haberse encontrado acabada. 
cuales coinciden con los contratos innominados del derecho común) $)^{3}$. A continuación debemos emplazar a Pufendorf (1632-1694) quien distingue directamente los contratos entre benéficos y onerosos ${ }^{4}$, no ya en la noción de acto, sino de contrato. Pero quien según sabemos, perfila la noción es Domat (1625-1696) para quien hay cuatro tipo de convenciones, que justamente parte de la denominación de los contratos innominados los cuales de sus distintos tipos indica los actos gratuitos ${ }^{5}$. Domat tanto al " $d o$ ut des", "facio ut facias" como al "facio ut des" las considera convenciones onerosas. Ahora bien, si sólo una de las partes da o hace algo, sin que el otro dé o haga significa que es una convención gratuita, y es así, como lo oneroso y gratuito queda perfilado en este autor ${ }^{6}$.

En el sistema contractual de Pothier, que, por lo demás, es similar a la clasificación que posteriormente se aprecia en Bello, se distingue contratos onerosos y gratuitos, además de los que denomina mixtos, utiliza la expresión "intéressés de part ét d'autre, de bienfaisance y mixtes". Y aun cuando intenta alejarse de la clasificación entre contratos nominados e innominados, de la misma manera que Domat, entiende que dicha clasificación "do ut des, facio et facias, facio ut des, do ut facias" correspondería a los contratos onerosos. Un tema que sí es relevante en Pothier es qué se entiende por interéressé ya que en esto es de lo que depende el concepto de lo oneroso y lo gratuito ya que como veremos Bello no utiliza dicho

${ }^{3}$ Ibíd., pp. 344-346. También puede aclarar más el panorama de la distinción entre contratos gratuitos y onerosos la obra de GuZMÁn BRITO, A., De las donaciones entre vivos, conceptos y tipos (Santiago de Chile, LexisNexis, 2005), pp. 148-152, al tratar sobre la liberalidad en Jean Domat.

${ }^{4}$ Ibíd., pp. 346-347. Guzmán entiende que de la lectura del De iure et nature et gentium, los contratos onerosos son divididos en los cuatro tipos de contratos innominados del Ius comunne: do ut des, do ut facias, facio ut des y facio ut facias.

${ }^{5}$ Ibíd., p. 347. Domat reenvía al lector al pasaje de Paulo D. 19,5,5 pr., (5, quaest.) : "Naturalis meus filius servit tibi et tuus filius mibi: convenit inter nos, ut et tu meum manumitteres et ego tuum: ego manumisi, tu non manumisisti: qua actione mibi teneris, quaesitum est. In hac quaestione totius ob rem dati tractatus inspici potest. Qui in his competit speciebus: aut enim do tibi ut des, aut do ut facias, aut facio ut des, aut facio ut facias: in quibus quaeritur, quae obligatio nascatur. [...]" ("Mi hijo natural te presta servidumbre y tu hijo a mí; se convino entre nosotros, que tu manumitieras al mío y yo al tuyo; yo lo manumití y tú no lo manumitiste; se preguntó qué acción me estarás obligado. En esta cuestión puede verse el tratado de todo lo que se da por causa de una cosa, el cual compete en estos casos. Porque o te doy para que des, o hagas, o hago para que des, o hago para que hagas; en cuyos casos se pregunta qué obligación nazca").

${ }^{6}$ Ibíd., pp. 347-348.

${ }^{7}$ Véase Pothier, Robert Joseph, Traité des obligations, párrs. 9-5, en Oeuvres de Pothier [...] X (edición de. Bugnet, 2a edición, Paris, 1861), X 
término sino otro cuando el contrato tiene por objeto "la utilidad" de una o ambas partes.

De todo lo anterior podemos concluir, con Guzmán, que la historia de la distinción entre contratos onerosos y gratuitos comenzó desde el iunaturalismo racionalista, probablemente ya incipiente en el humanismo jurídico ${ }^{8}$.

\section{Lo oneroso y lo gratuito en los proyectos del Código Civil de Chile.}

Si nos aproximamos a la historia del artículo 1440 CC. podremos apreciar que no muestra grandes variaciones en los diversos proyectos del mismo Código", sino sólo de índole meramente gramatical como es el cambio preposicional en la redacción: "a beneficio del otro" por "en beneficio". Observemos lo anterior, en cada uno de sus proyectos:

i) "Proyecto de 1842", título $1^{\circ}$, artículo 4: "El contrato es gratuito o de beneficencia, cuando solo tiene por objeto la utilidad de una de las partes, sufriendo la otra el gravámen; i oneroso, cuando tiene por objeto la utilidad de ambos contratantes, gravandose cada uno a beneficio del otro" 10 .

ii) "Proyecto de 1847", título $1^{\circ}$, artículo 4: "El contrato es gratuito o de beneficencia cuando solo tiene por objeto la utilidad de una de las partes, sufriendo la otra el gravamen; i oneroso, cuando tiene por objeto la utilidad de ambos contratantes, gravándose cada uno en beneficio del otro" ${ }^{11}$;

iii) "Proyecto de 1853", artículo 1617: "El contrato es gratuito o de beneficencia cuando solo tiene por objeto la utilidad de una de las partes, sufriendo la otra el gravamen; $i$ oneroso, cuando tiene por objeto la utilidad de ambos contratantes, gravándose cada uno a beneficio del otro"12;

iv) "Proyecto Inédito", artículo 1617: "El contrato es gratuito o de beneficencia cuando solo tiene por objeto la utilidad de una de las partes, sufriendo la otra el gravamen; i oneroso, cuando tiene por objeto la utilidad de ambos contratantes, gravándose cada uno a beneficio del otro"13.

${ }^{8}$ GuZmán Brito, Alejandro, Acto, negocio, contrato y causa, cit. (n. 1), p. 349.

${ }^{9}$ Guzmán Brito, Alejandro, El Primer Proyecto de Código Civil de Chile (Santiago, Editorial Jurídica de Chile, 1978); El Mismo, Estudios sobre los antecedentes sistemáticos de la parte general relativa a los actos y declaraciones de voluntad en el Código Civil de Chile y sus proyectos, en Revista de Estudios Histórico-Jurídicos, 2 (1977), pp. 20-22; El Mismo, Estudios de historia dogmática y sistemática sobre el código civil chileno-colombiano (Pontificia Universidad Javeriana, Bogotá, 2009), p. 268.

${ }^{10}$ Bello, Andrés, Obras completas, XI, pp. 141-142.

${ }^{11}$ Ibíd., XI, pp. 409-410.

${ }^{12}$ Ibíd., XII, p. 383.

${ }^{13}$ Ibíd., XIII, p. 365. 


\section{Lo oneroso y lo gratuito en los primeros códigos civiles.}

Sabemos que Bello no transcribió la redacción de los contratos gratuitos y onerosos del Code francés de $1804^{14}$. El cual, establece en sus artículos 1104 y 1105: i) "Le contrat de bienfaisance est celui dans lequel l'une des parties procure à l'autre un avantage purement gratuit"; y ii) "Le contrat à titre onereux est celui qui assujettit chacune des parties à donner ou à faire quelque chose". La redacción actual del Código Civil francés es la misma, el único cambio es que la materia está en los artículos 1105 y 1106.

Además del Code conocemos que Bello tuvo a la vista para la redacción de sus Proyectos de Código Civil en lo que atañe a los contratos onerosos y gratuitos, algunos que son de inspiración francesa, a saber, los códigos de Luisiana, de Cerdeña y del Reino de las Dos Sicilias.

i) El Código de la Luisiana acerca de la materia transcribe con exactitud el texto ya contenido en el artículo 5 CCFr.: "Le contrat de bienfaisance, est celui dans lequel une des parties procure à l'autre un avantage purement gratuit"; y en su artículo 6 : "Le contrat, à titre onéreux, est celui qui assujettit chacune des parties à donner ou à faire quelque chose”;

ii) El Código de la Cerdeña en un solo artículo, el 1192, trata ambos términos, pero continua con las mismas definiciones vistas en el francés. Así establece: "Quando ciascuna delle parti si assoggetta all'adempimento di qualche obbligazione, il contratto è a titolo oneroso. Quando una delle parti procura all'altra un vantaggio gratuito, il contratto è de beneficenza";

iii) En el Código del reino de las dos Sicilias nos encontramos que el artículo 1059 que define: "Il contratto di beneficenza, è quello in cui una delle parti procura all'altra un vantaggio meramente gratuito"; y el 1060: "Il contratto a titolo oneroso é quello che soggeta ciascuna delle parti a dare o a fare qualche cosa". La idea de utilidad o ventaja en un contrato oneroso viene a ser algo que la otra parte debe "dar" o "hacer", similar a lo que observamos en el francés: "donner ou à faire". En conclusión no podemos afirmar que Bello haya reproducido exacta la norma del artículo 1440 a partir de los códigos antes mencionados.

iv) En este rastreo de los primeros códigos no podemos dejar de mencionar al Código prusiano (ALPS), pues sabemos que nuestro código también tuvo una cierta influencia derivada del mismo. La materia es tratada en los siguientes parágrafos de la $1^{\text {a }}$ parte, título $5^{\circ}: \$ 7$ : “Wenn beide Theile gegenseitige Verbindlichkeiten übernehmen, so wird solches ein lästiger Vertrag genannt"; y $\$$ 8: “Wohlthätig heißt ein Vertrag, durch welchen nur Ein Theil etwas zu Gunsten des andern zu geben, zu leisten, zu dulden, oder zu unterlassen verpflichtet wird’. Lo importante en el $\$ 7$ es la idea de

\footnotetext{
${ }^{14}$ GuZmán Brito, A., Est. de hist dog. cit. (n. 9), 2009, p. 268.
} 
obligaciones recíprocas, más bien lo oneroso se parece a la definición de los contratos bilaterales del artículo $1439 \mathrm{CCCh}$. Y en cuanto a lo que indica el $\$ 8$ sobre el contrato "wohlthätig” ("benevolente”), la gratuidad está mirada no desde la perspectiva del que se beneficia, sino de aquel que soporta el gravamen de tolerar, permitir o abstenerse.

v) En el Código austríaco (ABGB) la materia es tratada en el $\$ 864$ : "Verträge sind einseitig oder zweiseitig verbindlich, je nachdem nur ein Theil etwas verspricht und der andere es annimmt; oder beide Theile einander Rechte übertragen, und wechselseitig annehmen. Die ersten werden also ohne Entgeld; die andern aber mit Entgeld geschlossen". Como podemos observar, este código identifica los contratos unilaterales ("einseitig") con los gratuitos o sin retribución ("ohne Entgeld"); y los bilaterales ("zweiseitig") con los onerosos o con retribución (mit Entgeld geschlossen).

\section{Lo oneroso y gratuito en Delvincourt.}

Delvincourt, en su obra Cours de Code Civil'15, presenta la distinción de "contrats de bienfaisance" y de "contrats à titre onéreux". Entiende que el primero también se denomina "à titre gratuit". Delvincourt dice que el contrato gratuito: "est celui qui n'a pour but que l'utilité de l'une des parties contractantes" y como ejemplo coloca el préstamo o mutuo sin interés, el depósito ${ }^{16}$ y el mandato; por contrato oneroso, que también denomina "intéressé de part et d'autre", entiende que "est celui qui a lieu pour l'intérêt et liutilité réciproque des parties, comme" y como ejemplos indica: el préstamo con interés, la compraventa ${ }^{17}$. Sabemos que Bello recogió tal clasificación y a su vez transcribió dichos conceptos ${ }^{18}$. La única diferencia es que Bello no hace alusión a los ejemplos que Delvincourt ofrece.

${ }^{15}$ Delvincourt, Claude Étienne, Cours de Code Civil, (2- edición, Bruselas, P.J. de Mat, 1827), I, pp. 79-80.

${ }^{16}$ Véase Delvincourt, C., cit. (n. 15), p. 79.

${ }^{17}$ Ibíd., "[...] définit le contrat à titre onéreux, celui qui assujétit chacune des parties à donner ou à faire quelque chose. Mais il est évident que cette définition, qui est réellement celle du contrat synallagmatique, ne peut s'appliquer au contrat à titre onéreux. Car, sous ce rapport, elle pèche contre une des règles de la logique, qui veut que toute définition convienne omni, et soli definito. Elle ne convient pas omni definito; car elle ne convient pas au prêt à intérêt, qui est certainement un contrat à titre onéreux, et dans lequel le prêteur n'est tenu de rien donner, ni de rien faire. Elle ne convient pas soli definito; car elle peut s'appliquer au dépôt, au mandat, qui sont des contrats de bienfaisance. [...]"; más adelante: "[...] Le contrat à titre onéreux est commutatif proiprement dit, ou aléatoire [...]",

${ }^{18}$ Véase GuZmán Brito, A., cit. (n. 1), pp. 343-354; El Mismo, cit. (n. 9), p. 268. 
A continuación veamos el estado de la cuestión acerca de los conceptos en la doctrina nacional.

\section{ESTADO DE LA CUESTIÓN SOBRE ALGUNOS PUNTOS EN LA DOCTRINA}

\section{CHILENA}

El examen del estado de la cuestión acerca de las categorías de lo oneroso y lo gratuito en la doctrina nacional revela varios problemas.

a) Gratuidad y donación. Un primer problema que se presenta en la determinación de lo gratuito es que suele identificarse esta categoría con la donación, porque ella resulta ser, en efecto, un paradigma de los actos gratuitos. Pero no es efectivo que deba extenderse las exigencias propias de la donación a otros actos gratuitos. Así, por ejemplo, como es necesario que en la donación siempre exista un enriquecimiento por una parte con la efectiva disminución del patrimonio por la otra, hay que determinar en qué casos ello no es exigible en actos gratuitos diferentes de la donación y por qué.

b) Gratuidad y lucratividad. Otro problema ${ }^{19}$, que aquí sólo enunciaremos, es el relativo a la significación de gratuidad y onerosidad frente al término "lucrativo". Lo anterior es debido a que nos encontramos en el Código Civil al menos con dos preceptos, los artículos 1962 inciso $1^{\circ}$ y 2303 , que utilizan tal expresión como sinónimos al título gratuito.

c) Sede de tratamiento. Es también problemática la sede en que

${ }^{19}$ Otro filón es aquel que dice relación con otras legislaciones del derecho privado; como por ejemplo, en materia de derecho comercial. Aquí, se menciona el carácter de oneroso o gratuito (sin referirse a lo lucrativo) en el libro IV: Reorganización y liquidación de empresas y personas deudoras, en sus artículos 133 (acerca del procedimiento concursal y de los bienes adquiridos a título gratuito y oneroso); 287 y 290 (de las acciones revocatorias en materia de los actos ejecutados o contratos suscritos por empresas deudoras y de los actos y contratos revocables celebrados por la persona deudora, respectivamente). Es interesante, pero en el derecho comercial se utilizan los términos onerosos y lucrativos como sinónimos, a diferencia del derecho civil, en que lo lucrativo equivale a lo gratuito. Además tenemos otras leyes sobre materias tales como: propiedad intelectual, rentas municipales, entre otras, que se refieren a los conceptos de onerosidad, gratuidad y lucratividad que debieran también ser revisados. Sin perjuicio de lo anterior adelantemos que por ejemplo, en el derecho español la gratuidad se confunde con la lucratividad. Sin embargo es posible proponer sobre la base del derecho romano una distinción de género a especie entre los actos a título gratuito y lucrativo, como lo ha habido un intento en los autores: VARAS, Juan Andrés, Anatomía del lucro: para una tipología jurídica, en Estudios de Derecho Civil. Jornadas Nacionales de Derecho Civil, Valdivia, 2013 (Santiago, LegalPublishing Thomson Reuters, 2014), IX, pp. 463-481; y Corral TalCiani, Hernán, en: https:// corraltalciani.wordpress.com/2013/10/13/lucro-y-titulo-lucrativo/. 
deba localizarse el tratamiento de nuestros conceptos, porque difiere entre los autores.

i) Una parte de la doctrina no hace alusión a ellos, ni en la teoría general de las obligaciones ni tampoco acerca de la teoría general de los contratos. Ésta se aprecia en autores como, Peñailillo, Ramos Pazos o Barcia Leh$\operatorname{mann}^{20}$. Los trata como parte de la teoría general de las fuentes de las obligaciones; así de Claro Solar y Abeliuk ${ }^{21}$. En cambio, Court, Domínguez, Pescio y Vial del Río ${ }^{22}$ exponen la distinción en materia de clasificación de los actos jurídicos. Otra tendencia la observamos en Ducci que se refiere al tema en la parte general del derecho civil; en la misma línea están Alessandri, Somarriva y Vodanovic ${ }^{23}$. Ahora bien, en Alessandri Rodríguez hallamos la distinción a propósito de la teoría general de los contratos al igual que lo hace López Santa María ${ }^{24}$. Y finalmente, nos encontramos con autores como Ruz Lártiga que se refieren a la distinción tanto en materia del acto jurídico ${ }^{25}$ como en la teoría general de los contratos.

ii) En cuanto a la extensión del tratamiento, podemos distinguir tres posiciones. La primera simplemente no expone la materia y ya fue señalada en el número anterior. La segunda tendencia es aquella en que la distin-

\footnotetext{
${ }^{20}$ Peñallillo Arévalo, Daniel, Obligaciones. Teoría general y clasificaciones. Resolución por incumplimiento (Santiago, Editorial jurídica de Chile, 2003); Ramos Pazos, René, De las obligaciones (3a edición, Santiago, LegalPublishing Chile, 2008); Barcia Lehmann, Rodrigo, Lecciones de derecho civil chileno: de las fuentes de las obligaciones (Santiago, Editorial Jurídica de Chile, 2007).

${ }^{21}$ Claro Solar, Luis, Explicaciones de derecho civil chileno y comparado (Santiago, Nascimento 1936), X, pp. 573-574; Abeliuk, René, Las obligaciones (5ª edición, Santiago, Editorial Jurídica de Chile, 2008), I, pp. 76-78.

${ }^{22}$ Court, Eduardo, Curso de derecho civil. Teoría general del acto jurídico (Santiago, LegalPublishing, 2009), p. 9; Domínguez, Ramón, Teoría general del negocio jurídico ( $2^{\mathrm{da}}$ edición, Editorial Jurídica de Chile, Santiago, 2012), pp. 27-28; PEscio, Victorio, Manual de derecho civil. Teoría de los actos jurídicos y teoría general de la prueba (Santiago, Editorial Jurídica de Chile, 1948), II, pp. 30-32; VIal DEL Río, V., cit. (n. 23), pp. 42-43.

${ }^{23}$ Ducci Claro, Carlos, Derecho civil: parte general (4 $4^{\mathrm{a}}$ edición, Santiago, Editorial Jurídica de Chile, 2009), pp. 238-239; Alessandri Rodríguez, Arturo - Somarriva Undurraga, Manuel - Vodanovic, Antonio, Tratado de derecho civil. Partes preliminar y general (7a edición, Santiago, Editorial Jurídica de Chile, 2005), II, p. 180.

${ }^{24}$ Alessandri Rodríguez, Arturo, De los contratos (Santiago, Editorial Jurídica de Chile, 2004), pp., 22-27; López Santa María, Jorge, Los contratos. Parte general (2a edición, Santiago, Editorial Jurídica de Chile, 1998), pp. 105-113.

${ }^{25}$ Ruz Lártiga, Gonzalo, Explicaciones de derecho civil. Parte general y acto jurídico (Santiago, LegalPublishing, 2011), en la parte general y acto jurídico, pp. 286-287 y en la teoría de los contratos, contratos y responsabilidad extracontractual, IV, pp. 37-38.
} 
ción entre lo gratuito y lo oneroso aparece al momento de tratar de las obligaciones en general o del acto jurídico, pero en forma concisa. Tal es el caso de Claro Solar, Pescio, Alessandri, Somarriva y Vodanovic, Ducci y Vial del Río ${ }^{26}$. Y una tercera postura es la que observamos en autores como Abeliuk, Alessandri Rodríguez, Domínguez y López Santa María ${ }^{27}$, quienes tratan la materia en forma más extensa.

iii) Los autores divergen acerca de la naturaleza de la gratuidad y la onerosidad, en cuanto basada en la "utilidad". Algunos, como Abeliuk, Alessandri y Domínguez ${ }^{28}$, expresan que la utilidad es un gravamen o bien una ventaja, que puede ser incluso meramente moral, esto es, sin un valor pecuniario o material. Este último autor, indica que para determinar si un acto es de comercio o es un contrato se necesita que exista un fin de lucro, por lo que pareciera que este último se identificaría con el carácter de oneroso. A su vez, López Santa María señala que lo que distingue a los contratos gratuitos y onerosos es un criterio netamente económico. Cabe añadir que las posturas entre lo subjetivo y objetivo a propósito de la onerosidad también las menciona Domínguez.

En cambio encontramos la postura de Guzmán Brito ${ }^{29}$ que al momento de considerar la gratuidad tanto de los legados como de las donaciones entre vivos, explica que lo que delimita la onerosidad de la gratuidad es el precio, sin confundirlo con el dinero. Por tanto, la onerosidad implica un elemento objetivo: el precio, a diferencia de lo sostenido por los autores anteriores que recurren al elemento moral o subjetivo ${ }^{30}$.

${ }^{26}$ Claro Solar, L., cit. (n. 21), pp. 573-574; Pescio,V., cit. (n. 22), pp. 30-32; Alessandri - Somarriva - Vodanovic, cit. (n. 18), p. 180; Ducci., C., cit. (n. 23), pp. 238-239; Vial del Río, V., cit. (n. 23), pp. 42-43.

${ }^{27}$ Abeliuk, R., cit. (n. 21), pp. 76-82; Alessandri Rodríguez, A., cit. (n. 24), pp., 22-27; Domínguez, R., cit. (n. 22), pp. 27-28 y López Santa María, J., cit. (n. 24), pp. 105-113.

${ }^{28}$ Abeliuk, René, cit. (. 21), p. 78; Alessandri Rodríguez, Arturo - Somarriva Undurraga, Manuel - Vodanovic, Antonio, Tratado de derecho civil. Partes preliminar y general (7a edición, Editorial Jurídica de Chile, Santiago, 2005), II, p. 25 y Domínguez, Ramón, cit. (n. 22), p. 27. Entre estos autores Alessandri insiste en la noción de la utilidad como un gravamen.

${ }^{29}$ GuZmán Brito, A., Est., sobre legados., cit. (n. 21), pp. 28-43; El Mismo, De las donaciones entre vivos, conceptos y tipos (cit. (n. 3), pp. 41-42.

${ }^{30}$ Ibíd., define claramente el elemento lucrativo, como "[...] el fenómeno consistente en que el destinatario de la atribución patrimonial consiga el objeto de ésta sin correlativamente quedar sujeto a restituirlo con posterioridad, de modo de poder retenerlo" (2005), p. 49, esto es, el carácter definitivo de una adquisición patrimonial (2012), pp. 37-43, en consecuencia en ninguna de las mencionadas liberalidades existe una obligación de restitución de lo donado o legado. También aclara que no se puede confundir "[...] la lucratividad en que consiste una donación con el enri- 
iv) Pescio es el único autor que aplica la noción de gratuidad al acto jurídico, pues define al acto gratuito como el "inspirado o determinado por un exclusivo propósito de beneficencia (benefacere, hacer el bien) [...]"31 y da como ejemplo, el testamento.

Debido a que el paradigma de este tipo de contratos es por excelencia la donación, los autores, al momento de explicar este carácter, recurren a ella y exigen que la parte que soporta el gravamen necesariamente tenga una disminución patrimonial, denominada empobrecimiento del donante y un aumento para el donatario, esto que se ha denominado enriquecimiento del donatario, ambos requisitos exigidos por el artículo 1398 CC., para la donación. Por ende, el problema es saber si siempre que existe gratuidad, necesariamente deberá exigirse el aumento y disminución patrimonial de las partes que intervienen en el negocio, aplicando las normas que rigen la donación, debido a que éste es el negocio que siempre se menciona.

v) La importancia de distinguir entre actos gratuitos $\mathrm{u}$ onerosos se encuentra en los efectos prácticos que ésta produce. En este aspecto, sólo el siguiente elenco de autores los expone como son Abeliuk, Alessandri, Domínguez, López Santa María, Pescio y Ruz Lártiga; entre ellos hay una cierta unanimidad respecto a algunos efectos y otros sólo son enunciados por alguno o algunos.

d) Discriminación de efectos. En cuanto a los efectos que importa la distinción entre la onerosidad y la gratuidad, la doctrina ha abordado algunos, pero nos parece que podrían ser tratados con mayor profundidad, y verificar, además, que se presentan en otros que escapan a la contractualidad y pertenecen a la negociabilidad en general. Pensamos que es posible construir una delimitación dinámica de la gratuidad/onerosidad, que vaya en función de los efectos que se piensa obtener de esa calificación.

Es así como los efectos que enuncian son:

i) Hay una mayor restricción en cuanto a las liberalidades, como lo indican Abeliuk, Domínguez, López Santa María y Pescio ${ }^{32}$. Como lo es en materia de donaciones, que son restringidas por el posible perjuicio que puede haber en el futuro para asignatarios forzosos del donante. Esto es

quecimiento que ella suele provocar [...]" lo que explicita con el ejemplo de donar 1.000, para que el donatario dé los 1.000 a un tercero. En este caso hay lucratividad, pero no hay enriquecimiento, debido a que tendrá que dar los 1.000. Su patrimonio queda de la misma forma que antes de recibir la donación. Pero los adquirió de manera definitiva, esto es, hubo atribución patrimonial, que es el carácter lucrativo.

${ }^{31}$ Pescio, V., cit. (n. 22), pp. 30-31.

${ }^{32}$ Abeliuk, R., cit. (n. 21), p. 81; Domínguez, R., cit. (n. 22), pp. 27-28; López Santa María, J., cit. (n. 20), pp. 111-112; Pescio, V., cit. (n. 22), p. 31. 
visible, ya que se necesita la insinuación para las donaciones irrevocables y la obtención de autorización judicial.

ii) El error en la persona vicia el consentimiento en los contratos a título gratuito; esto es sostenido por Abeliuk, Alessandri, López Santa María, Pescio y Ruz Lártiga ${ }^{33}$.

iii) Para determinar el tipo de culpa por la cual debe responder el deudor, cobra relevancia si el acto es gratuito u oneroso. Esto lo mencionan en sus exposiciones Abeliuk, Alessandri, Claro Solar, López Santa María, Pescio y Ruz Lártiga ${ }^{34}$.

iv) Para determinar la procedencia de la acción pauliana en conformidad con el artículo 2468 CC., también es importante la distinción que nos ocupa, como lo indican Abeliuk, Alessandri, López Santa María, Pescio y Ruz Lártiga ${ }^{35}$.

v) La obligación de garantizar la evicción no se aplica a los negocios a título gratuito, sí en cambio para los onerosos, según lo sostienen Abeliuk, Alessandri, López Santa María y Ruz Lártiga ${ }^{36}$.

vi) Para ejercer por terceros la acción del pago de lo "no debido" el legislador establece en el artículo 2303 CC. que debe estarse a la distinción entre actos a título gratuito u oneroso. Quienes lo señalan son Abeliuk, López Santa María y Ruz Lártiga ${ }^{37}$.

vii) Tanto Alessandri como Pescio ${ }^{38}$ consideran otras dos materias en las que la distinción entre contratos gratuitos y onerosos es relevante: $i$ ) para determinar la transmisibilidad de los derechos del acreedor condicional que fallece entre el contrato y el cumplimiento de la condición; y ii) Para establecer el carácter civil o mercantil de un contrato indican que los contratos gratuitos no son nunca comerciales. Es sobre este tema, como ya dijimos, donde Alessandri dice que para que exista un acto de comercio se requiere siempre un fin de lucro como oneroso.

${ }^{33}$ Abeliuk, R., cit. (n. 21), p. 82; Alessandri, A., cit. (n. 24), p, 26; López Santa María, J., cit. (n. 24), p. 111; Pescio, V., cit. (n. 22), p. 31; Ruz lártiga, G., cit. (n. 25), p. 37.

${ }^{34}$ Abeliuk, R., cit. (n. 21), p. 82; Ibíd, Alessandri, A., p. 26; López Santa MaRía, cit. (n. 24), p. 110; Claro Solar, L., cit. (n. 21), p. 574; Varas, J., cit. (n. 25), pp. 463-481.

${ }^{35}$ Abeliuk, R., cit. (n. 21), pp. 26-27; López Santa María, J., cit. (n. 24), p. 111; Ruz LÁrtiga, G., cit. (n. 25), p. 38; 2, V., cit. (n. 23), p. 31;

${ }^{36}$ Abeliuk, R., cit. (n. 21), Alessandri, A., cit. (n. 24), p. 26, López Santa MaRíA, J., cit. (n. 24), p. 110; RUZ LÁrTiga, G., cit. (n. 25), p. 37.

${ }^{37}$ Abeliuk, R., cit. (n. 21); López Santa María, J., cit. (n. 24), p. 112, Ruz LárTIGA, G., cit. (n. 25), p. 38.

${ }^{38}$ Alessandri, A., cit. (n. 24), p. 27; Pescio, V., cit. (n. 22), pp. 31-32. 
viii) López Santa María ${ }^{39}$ añade que debe existir un reconocimiento por parte del que es beneficiado con un contrato gratuito hacia aquel que lo beneficia. Esto principalmente acaece en materia de donación.

\section{CONCLUSIONES}

De acuerdo a lo indicado al iniciar esta investigación, el lector ha de tener en cuenta que nuestra investigación está en sus comienzos. Con todo podemos afirmar que:

$1^{\circ}$ La historia de la distinción entre lo oneroso y lo gratuito se generó a través del desarrollo del iusnaturalismo racionalista, probablemente ya incipiente en el humanismo jurídico.

$2^{\circ}$ De la comparación de las codificaciones, vemos que el artículo 1440 CC. guarda una cierta semejanza con el Code francés de 1804 en los artículos 1104 y 1105. Pero de quien se sabe que Bello definitivamente transcribió las nociones en estudio fue del Cours de Code Civil de Claude Delvincourt; en embargo, la redacción del artículo 1440 no recogió los ejemplos que este autor indica en su obra, a pesar que en varias partes del Código de Bello, sí existen ejemplos en diferentes instituciones.

iii) Respecto a la doctrina nacional acerca de lo oneroso y gratuito indicamos que el término de "utilidad" no es comprendido de la misma manera por todos. Hay un énfasis en lo subjetivo, a veces, y en lo objetivo en otros. Es por ello que queda pendiente para la continuación de esta investigación llegar a dicha noción, principalmente a través de la jurispudencia.

iv) $\mathrm{Y}$, por último, también en relación con la doctrina chilena, la sede donde es localizada como también las consecuencias civiles que acarrea la distinción en diversos ámbitos, nos parece que no es conteste, de allí a la necesidad de una revisión tanto legal como jurisprudencial de sus mismos efectos.

\section{BIBLIOGRAFÍA}

Abeliuk Manasevich, René, Las obligaciones, I (5ª edición, Santiago, Editorial Jurídica de Chile, 2008), I.

Alessandri Rodríguez, Arturo, De los contratos (Santiago, Editorial Jurídica de Chile, 2004).

Alessandri Rodríguez, Arturo - Somarriva Undurraga, Manuel - Vodanovic, Antonio, Tratado de derecho civil. Partes preliminar y general (7a edición, Editorial Jurídica de Chile, Santiago, 2005), II.

ARCHI, Gian Gualberto, La donazione. Corso di diritto romano (Milano, Giuffrè, 1960).

${ }^{39}$ López Santa María, J., cit. (n. 24), p. 111. 
Barcia Lehmann, Rodrigo, Lecciones de derecho civil chileno: de las fuentes de las obligaciones (Santiago, Editorial Jurídica de Chile, 2007).

Bello, Andrés, Obras completas de don Andrés Bello (Santiago de Chile, Pedro G. Ramírez, 1887), XI.

Bello, Andrés, Obras Completas de don Andrés Bello (Santiago de Chile, Pedro G. Ramírez, 1888), XII.

Benabént, Alain, Droit civil. Les obligations (Paris, 2007).

Bianca, Massimo, Diritto civile (Milano, Giuffrè, 1998).

Capitant, Henri, De la cause des obligations (2a edición, Dalloz, Paris, 1924).

Claro Solar, Luis, Explicaciones de derecho civil chileno y comparado (Santiago, Nascimento 1936), X.

Code Civil des Français (ed. facsimilar de la original de 1804, Dalloz, 2004).

Corral Talciani, Hernán en: https://corraltalciani.wordpress.com/2013/10/13/ lucro-y-titulo-lucrativo/.

Court, Eduardo, Curso de derecho civil teoría general del acto jurídico (Santiago de Chile, LegalPublishing, 2009).

De Prada González, José María, La onerosidad y gratuidad de los actos jurídicos, en Anales de la Academia Matritense del Notariado, 16 (Madrid, 1968).

Delvincourt, Claude-Étienne, Cours de Code Civil (2 ${ }^{\mathrm{da}}$ edición, Bruxeless, P.J. de Mat, 1827).

Díez-PicAzo, Luis - Gullón, Antonio, Instituciones de derecho civil: doctrina general del contrato y de las obligaciones (2a edición, Madrid, Tecnos, 1998), I,2.

Díez-Picazo, Luis, Fundamentos del derecho civil patrimonial: introducción teoría del contrato (6a edición, Civitas, 2007), I,2.

Domínguez, Ramón, Teoría general del negocio jurídico ( $2^{\mathrm{da}}$ edición, Editorial Jurídica de Chile, Santiago, 2012).

Ducci Claro, Carlos, Derecho civil: parte general (4 edición, Santiago, Editorial Jurídica de Chile, 2009).

Dupeyroux, Jean-Jacques, Contribution a la théorie générale de l'acte a titre gratuit (Paris, Librairie Générale de Droit et de Jurisprudence, 1955).

Galgano, Francesco, Trattato di diritto civile (2 ${ }^{\mathrm{da}}$ edición, Padova, CEDAM, 2010), II.

GuZmán Brito, Alejandro, Acto, negocio, contrato y causa en la tradición del derecho europeo e iberoamericano (Navarra, Thomson - Aranzadi, 2005), pp. 11-196.

GuZMán Brito, Alejandro, Andrés Bello codificador. Historia de la fijación y codificación del derecho civil en Chile (Santiago, Ediciones Universidad de Chile, 1982), I.

GuZMÁn Brito, Alejandro, De las donaciones entre vivos, conceptos y tipos (Santiago, LexisNexis, 2005).

Guzmán Brito, Alejandro, El Primer Proyecto de Código Civil de Chile. Estudio histórico-crítico introductorio y reedición del proyecto (Santiago, Editorial Jurídica de Chile, 1978).

GuZMÁn BRITO, Alejandro, Estudio sobre los antecedentes sistemáticos de la parte general relativa a los actos y declaraciones de voluntad en el Código Civil de Chile y sus proyectos, en Revista de Estudios Histórico-Jurídicos, 2 (1977).

GuZMÁN BRITO, Alejandro, Estudios de historia dogmática y sistemática sobre el código civil chileno colombiano (Bogotá, Pontificia Universidad Javeriana, 2009).

GuZMÁn BRITO, Alejandro, Estudios sobre los legados según el derecho civil chileno (Santiago, LegalPublishing, 2012). 
López Santa María, Jorge, Los contratos. Parte general (2a edición, Santiago, Editorial Jurídica de Chile, 1998).

Michel, Jacques, Gratuité en droit romain (Bruxelles, Universitè libre de Bruxelles Institut de Sociologie, 1962).

Palermo, Antonio, s. v. “Onere”, en Novissimo Digesto Italiano, XI ( $3^{\text {a }}$ edición, Torino, Editricie Torinense, 1965).

Peñailillo Arévalo, Daniel, Obligaciones. Teoría general y clasificaciones. Resolución por incumplimiento (Santiago, Editorial jurídica de Chile, 2003).

Pescio, Victorio, Manual de derecho civil Teoría de los actos jurídicos y teoría general de la prueba (Santiago, Editorial Jurídica de Chile, 1948), II.

Planiol, Marcel - Ripert, Georges, Traité pratique de droit civil français, VI: Obligations (Paris, 1930).

Pothier, Robert-Joseph, Traité des obligations, en Oeuvres de Pothier (edición de M. Bugnet, 2a edición, París, 1861), X.

Puig Peña, Federico, Tratado de derecho civil español (Madrid, Editorial Revista de Derecho Privado, 1946), IV, 1.

Ramos Pazos, René, De las obligaciones (3a edición, Santiago, LegalPublishing, 2008).

Revuelta Salazar, María, La gratuidad del “mutuum” en el derecho romano (Jaén, Servicio de Publicaciones de la Universidad de Jaén, 1999).

Ruz Lártiga, Gonzalo, Explicaciones de derecho civil. Parte general y acto jurídico (Santiago, LegalPublishing, 2011.

SCHIPANI, Sandro, Del derecho romano a las codificaciones latinoamericanas. La obra de Andrés Bello. Pluralidad de fuentes del tít. I del Lib. IV del Código Civil de Chile $y$ de sus proyectos en Revista de Estudios Histórico-Jurídicos, 6 (Valparaíso, 1981).

VARAS, Juan Andrés, Anatomía del lucro: para una tipología jurídica, en Estudios de Derecho Civil. Jornadas Nacionales de Derecho Civil, Valdivia, 2013 (Santiago, LegalPublishing - Thomson Reuters, 2014), IX.

VIAL DEL Río, Víctor, Teoría general del acto jurídico (5ª edición, Santiago, Editorial Jurídica de Chile, 2006). 